\title{
Blow-Up Phenomena for Nonlinear Reaction-Diffusion Equations under Nonlinear Boundary Conditions
}

\author{
Juntang Ding \\ School of Mathematical Sciences, Shanxi University, Taiyuan 030006, China \\ Correspondence should be addressed to Juntang Ding; djuntang@sxu.edu.cn
}

Received 20 November 2015; Accepted 6 March 2016

Academic Editor: Leszek Olszowy

Copyright (C) 2016 Juntang Ding. This is an open access article distributed under the Creative Commons Attribution License, which permits unrestricted use, distribution, and reproduction in any medium, provided the original work is properly cited.

\begin{abstract}
This paper deals with blow-up and global solutions of the following nonlinear reaction-diffusion equations under nonlinear boundary conditions: $(g(u))_{t}=\nabla \cdot(a(u) \nabla u)+f(u)$ in $\Omega \times(0, T), \partial u / \partial n=b(x, u, t)$ on $\partial \Omega \times(0, T), u(x, 0)=u_{0}(x)>0$, in $\bar{\Omega}$, where $\Omega \subset \mathbb{R}^{N}(N \geq 2)$ is a bounded domain with smooth boundary $\partial \Omega$. We obtain the conditions under which the solutions either exist globally or blow up in a finite time by constructing auxiliary functions and using maximum principles. Moreover, the upper estimates of the "blow-up time," the "blow-up rate," and the global solutions are also given.
\end{abstract}

\section{Introduction}

During the past few decades, the blow-up phenomena for the nonlinear reaction-diffusion equations have been studied by a large number of authors, and the reader is referred to [1-8] and the references therein. In this paper, we consider the following nonlinear reaction-diffusion problem under nonlinear conditions:

$$
\begin{aligned}
(g(u))_{t} & =\nabla \cdot(a(u) \nabla u)+f(u) \quad \text { in } \Omega \times(0, T), \\
\frac{\partial u}{\partial n} & =b(x, u, t) \quad \text { on } \partial \Omega \times(0, T), \\
u(x, 0) & =u_{0}(x)>0 \quad \text { in } \bar{\Omega},
\end{aligned}
$$

where $\Omega \subset \mathbb{R}^{N}(N \geq 2)$ is a bounded domain with smooth boundary $\partial \Omega, \partial u / \partial n$ represents the outward normal derivative on $\partial \Omega$, and $T$ is the maximal existence time of $u$. Set $\mathbb{R}_{+}:=(0,+\infty)$. We assume, throughout the paper, that $a(s)$ is a positive $C^{2}\left(\mathbb{R}_{+}\right)$function, $g(s)$ is a $C^{2}\left(\mathbb{R}_{+}\right)$ function, $g^{\prime}(s)>0$ for any $s \in \mathbb{R}_{+}, f(s)$ is a nonnegative $C^{1}\left(\mathbb{R}_{+}\right)$function, $b(x, s, t)$ is a nonnegative $C^{1}\left(\partial \Omega \times \mathbb{R}_{+} \times \mathbb{R}_{+}\right)$ function, and $u_{0}(x)$ is a positive $C^{2}\left(\overline{\mathbb{R}_{+}}\right)$function and satisfies the compatibility conditions. Under the above assumptions, the local existence and uniqueness of classical solution of problem (1) were established by Amann [9]. Furthermore, it follows from maximum principle [10] and regularity theorem
[11] that the solution $u(x, t)$ is positive and $u(x, t) \in C^{3}(\Omega \times$ $(0, T)) \cap C^{2}(\bar{\Omega} \times(0, T))$.

Many authors have investigated blow-up and global solutions of nonlinear reaction-diffusion equations under nonlinear boundary conditions and have obtained a lot of interesting results (see, e.g., [12-20]). To my knowledge, some special cases of (1) have been studied. Zhang [21] considered the following problem:

$$
\begin{aligned}
(g(u))_{t} & =\Delta u+f(u) \quad \text { in } \Omega \times(0, T), \\
\frac{\partial u}{\partial n} & =b(u) \quad \text { on } \partial \Omega \times(0, T) \\
u(x, 0) & =u_{0}(x)>0 \quad \text { in } \bar{\Omega}
\end{aligned}
$$

where $\Omega \subset \mathbb{R}^{N}(N \geq 2)$ is a bounded domain with smooth boundary $\partial \Omega$. By constructing auxiliary functions and using maximum principles, the existence of blow-up and global solutions were obtained under appropriate assumptions on the functions $b, f, g$, and $u_{0}$. Zhang et al. [22] dealt with the following problem:

$$
\begin{aligned}
(g(u))_{t} & =\nabla \cdot(a(u) \nabla u)+f(u) \quad \text { in } \Omega \times(0, T), \\
\frac{\partial u}{\partial n} & =b(u) \quad \text { on } \partial \Omega \times(0, T), \\
u(x, 0) & =u_{0}(x)>0 \quad \text { in } \bar{\Omega},
\end{aligned}
$$


where $\Omega \subset \mathbb{R}^{N}(N \geq 2)$ is a bounded domain with smooth boundary $\partial \Omega$. Some conditions on nonlinearities and the initial data were given to ensure that $u(x, t)$ exists globally or blows up at some finite time $T$. In addition, the upper estimates of the global solution, the "blow-up time," and the "blow-up rate" were also established.

In this paper, we study reaction-diffusion problem (1). It is well known that $f(u), g(u), a(u)$, and $b(x, u, t)$ are nonlinear reaction, nonlinear diffusion, nonlinear convection, and nonlinear boundary flux, respectively. What interactions among the four nonlinear mechanisms result in the blow-up and global solutions of (1) is investigated in this work. We note that the boundary flux function $b(x, u, t)$ depends not only on the concentration variable $u$ but also on the space variable $x$ and the time variable $t$. Hence, it seems that the methods of $[21,22]$ are not applicable for problem (1). In this paper, by constructing completely different auxiliary functions from those in $[21,22]$ and technically using maximum principles, we obtain the existence theorems of the blow-up and global solution. Moreover, the upper estimates of "blow-up time," "blow-up rate," and global solution are also given. Our results can be seen as the extension and supplement of those obtained in $[21,22]$.

The present work is organized as follows. In Section 2, we deal with the blow-up solution of (1). Section 3 is devoted to the global solution of (1). As applications of the obtained results, some examples are presented in Section 4.

\section{Blow-Up Solution}

In this section, we discuss what interactions among the four nonlinear mechanisms of (1) result in the blow-up solution. Our main result in this section is the following theorem.

Theorem 1. Let $u(x, t)$ be a solution of problem (1). Assume that the following conditions (i)-(iv) are satisfied.

(i) For $s \in \mathbb{R}_{+}$,

$$
\begin{gathered}
a(s)-a^{\prime}(s) \geq 0, \\
f^{\prime}(s)-f(s) \geq 0, \\
\left(\frac{a(s)}{g^{\prime}(s)}\right)^{\prime} \geq 0 .
\end{gathered}
$$

(ii) For $(x, s, t) \in \partial \Omega \times \mathbb{R}_{+} \times \mathbb{R}_{+}$,

$$
\begin{aligned}
b_{s}(x, s, t)-b(x, s, t) & \leq 0, \\
a(s) b(x, s, t)-(a(s) b(x, s, t))_{s} & \leq 0, \\
b_{t}(x, s, t) & \geq 0 .
\end{aligned}
$$

(iii) Consider the following:

$$
\alpha:=\min _{\bar{D}}\left\{\frac{a\left(u_{0}\right)\left[\nabla \cdot\left(a\left(u_{0}\right) \nabla u_{0}\right)+f\left(u_{0}\right)\right]}{\mathrm{e}^{u_{0}} g^{\prime}\left(u_{0}\right)}\right\}>0 .
$$

(iv) Consider the following:

$$
\int_{M_{0}}^{+\infty} \frac{a(s)}{\mathrm{e}^{s}} \mathrm{~d} s<+\infty, \quad M_{0}:=\max _{\bar{D}} u_{0}(x) .
$$

Then $u(x, t)$ blows up in a finite time $T$ and

$$
\begin{array}{r}
T \leq \frac{1}{\alpha} \int_{M_{0}}^{+\infty} \frac{a(s)}{\mathrm{e}^{s}} \mathrm{~d} s, \\
u(x, t) \leq H^{-1}(\alpha(T-t)),
\end{array}
$$

where

$$
H(y):=\int_{y}^{+\infty} \frac{a(s)}{\mathrm{e}^{s}} \mathrm{~d} s, \quad y>0,
$$

and $\mathrm{H}^{-1}$ is the inverse function of $\mathrm{H}$.

Proof. Introduce an auxiliary function

$$
P(x, t):=-\mathrm{e}^{-u} u_{t}+\alpha \frac{1}{a(u)},
$$

and then we have

$$
\begin{aligned}
\nabla P= & \mathrm{e}^{-u} u_{t} \nabla u-\mathrm{e}^{-u} \nabla u_{t}-\alpha \frac{a^{\prime}}{a^{2}} \nabla u, \\
\Delta P= & -\mathrm{e}^{-u} u_{t}|\nabla u|^{2}+2 \mathrm{e}^{-u} \nabla u \cdot \nabla u_{t}+\mathrm{e}^{-u} u_{t} \Delta u \\
& -\mathrm{e}^{-u} \Delta u_{t}+\left(2 \alpha \frac{\left(a^{\prime}\right)^{2}}{a^{3}}-\alpha \frac{a^{\prime \prime}}{a^{2}}\right)|\nabla u|^{2} \\
& -\alpha \frac{a^{\prime}}{a^{2}} \Delta u, \\
P_{t}= & \mathrm{e}^{-u}\left(u_{t}\right)^{2}-\mathrm{e}^{-u}\left(u_{t}\right)_{t}-\alpha \frac{a^{\prime}}{a^{2}} u_{t} \\
= & \mathrm{e}^{-u}\left(u_{t}\right)^{2}-\mathrm{e}^{-u}\left(\frac{a}{g^{\prime}} \Delta u+\frac{a^{\prime}}{g^{\prime}}|\nabla u|^{2}+\frac{f}{g^{\prime}}\right)_{t} \\
& -\alpha \frac{a^{\prime}}{a^{2}} u_{t} \\
= & \mathrm{e}^{-u}\left(u_{t}\right)^{2}+\left(\frac{a g^{\prime \prime}}{\left(g^{\prime}\right)^{2}}-\frac{a^{\prime}}{g^{\prime}}\right) \mathrm{e}^{-u} u_{t} \Delta u \\
& -\frac{a}{g^{\prime}} \mathrm{e}^{-u} \Delta u_{t}+\left(\frac{a^{\prime} g^{\prime \prime}}{\left(g^{\prime}\right)^{2}}-\frac{a^{\prime \prime}}{g^{\prime}}\right) \mathrm{e}^{-u} u_{t}|\nabla u|^{2} \\
& -2 \frac{a^{\prime}}{g^{\prime}} \mathrm{e}^{-u}\left(\nabla u \cdot \nabla u_{t}\right)+\left(\frac{f g^{\prime \prime}}{\left(g^{\prime}\right)^{2}}-\frac{f^{\prime}}{g^{\prime}}\right) \mathrm{e}^{-u} u_{t} \\
& -\alpha \frac{a^{\prime}}{a^{2}} u_{t} .
\end{aligned}
$$


It follows from (12) and (13) that

$$
\begin{aligned}
\frac{a}{g^{\prime}} \Delta P-P_{t}= & \left(\frac{a^{\prime \prime}}{g^{\prime}}-\frac{a}{g^{\prime}}-\frac{a^{\prime} g^{\prime \prime}}{\left(g^{\prime}\right)^{2}}\right) \mathrm{e}^{-u} u_{t}|\nabla u|^{2} \\
& +\left(2 \frac{a}{g^{\prime}}+2 \frac{a^{\prime}}{g^{\prime}}\right) \mathrm{e}^{-u}\left(\nabla u \cdot \nabla u_{t}\right) \\
& +\left(\frac{a^{\prime}}{g^{\prime}}+\frac{a}{g^{\prime}}-\frac{a g^{\prime \prime}}{\left(g^{\prime}\right)^{2}}\right) \mathrm{e}^{-u} u_{t} \Delta u \\
& +\left(2 \alpha \frac{\left(a^{\prime}\right)^{2}}{a^{2} g^{\prime}}-\alpha \frac{a^{\prime \prime}}{a g^{\prime}}\right)|\nabla u|^{2} \\
& -\alpha \frac{a^{\prime}}{a g^{\prime}} \Delta u-\mathrm{e}^{-u}\left(u_{t}\right)^{2} \\
& +\left(\frac{f^{\prime}}{g^{\prime}}-\frac{f g^{\prime \prime}}{\left(g^{\prime}\right)^{2}}\right) \mathrm{e}^{-u} u_{t}+\alpha \frac{a^{\prime}}{a^{2}} u_{t} .
\end{aligned}
$$

The first equation of (1) implies

$$
\Delta u=\frac{g^{\prime}}{a} u_{t}-\frac{a^{\prime}}{a}|\nabla u|^{2}-\frac{f}{a} .
$$

Inserting (15) into (14), we obtain

$$
\begin{aligned}
\frac{a}{g^{\prime}} \Delta P-P_{t}= & \left(\frac{a^{\prime \prime}}{g^{\prime}}-\frac{a}{g^{\prime}}-\frac{\left(a^{\prime}\right)^{2}}{a g^{\prime}}-\frac{a^{\prime}}{g^{\prime}}\right) \mathrm{e}^{-u} u_{t}|\nabla u|^{2} \\
& +\left(2 \frac{a}{g^{\prime}}+2 \frac{a^{\prime}}{g^{\prime}}\right) \mathrm{e}^{-u}\left(\nabla u \cdot \nabla u_{t}\right) \\
& +\frac{g^{\prime}}{a}\left(\frac{a}{g^{\prime}}\right)^{\prime} \mathrm{e}^{-u}\left(u_{t}\right)^{2} \\
& +\left(\frac{f^{\prime}}{g^{\prime}}-\frac{a^{\prime} f}{a g^{\prime}}-\frac{f}{g^{\prime}}\right) \mathrm{e}^{-u} u_{t} \\
& +\left(3 \alpha \frac{\left(a^{\prime}\right)^{2}}{a^{2} g^{\prime}}-\alpha \frac{a^{\prime \prime}}{a g^{\prime}}\right)|\nabla u|^{2}+\alpha \frac{a^{\prime} f}{a^{2} g^{\prime}}
\end{aligned}
$$

It follows from (11) that

$$
\nabla u_{t}=-\mathrm{e}^{u} \nabla P+u_{t} \nabla u-\alpha \frac{a^{\prime}}{a^{2}} \mathrm{e}^{u} \nabla u
$$

Substituting (17) into (16), we get

$$
\begin{aligned}
\frac{a}{g^{\prime}} \Delta P & +2 \frac{a+a^{\prime}}{g^{\prime}} \nabla u \cdot \nabla P-P_{t} \\
= & \left(\frac{a^{\prime \prime}}{g^{\prime}}+\frac{a}{g^{\prime}}-\frac{\left(a^{\prime}\right)^{2}}{a g^{\prime}}+\frac{a^{\prime}}{g^{\prime}}\right) \mathrm{e}^{-u} u_{t}|\nabla u|^{2} \\
& +\left(\alpha \frac{\left(a^{\prime}\right)^{2}}{a^{2} g^{\prime}}-2 \alpha \frac{a^{\prime}}{a g^{\prime}}-\alpha \frac{a^{\prime \prime}}{a g^{\prime}}\right)|\nabla u|^{2} \\
& +\frac{g^{\prime}}{a}\left(\frac{a}{g^{\prime}}\right)^{\prime} \mathrm{e}^{-u}\left(u_{t}\right)^{2} \\
& +\left(\frac{f^{\prime}}{g^{\prime}}-\frac{a^{\prime} f}{a g^{\prime}}-\frac{f}{g^{\prime}}\right) \mathrm{e}^{-u} u_{t}+\alpha \frac{a^{\prime} f}{a^{2} g^{\prime}} .
\end{aligned}
$$

By (10), we have

$$
u_{t}=-\mathrm{e}^{u} P+\alpha \mathrm{e}^{u} \frac{1}{a}
$$

Now, we insert (19) into (18) to deduce

$$
\begin{aligned}
\frac{a}{g^{\prime}} \Delta P & +2 \frac{a^{\prime}+a}{g^{\prime}} \nabla u \cdot \nabla P \\
& +\frac{a}{g^{\prime}}\left\{\left[1+\frac{a^{\prime}}{a}+\left(\frac{a^{\prime}}{a}\right)^{\prime}\right]|\nabla u|^{2}+\left(\frac{f}{a}\right)^{\prime}-\frac{f}{a}\right\} P \\
& -P_{t} \\
= & \alpha \frac{1}{a g^{\prime}}\left(a-a^{\prime}\right)|\nabla u|^{2}+\frac{g^{\prime}}{a}\left(\frac{a}{g^{\prime}}\right)^{\prime} \mathrm{e}^{-u}\left(u_{t}\right)^{2} \\
& +\alpha \frac{1}{a g^{\prime}}\left(f^{\prime}-f\right) .
\end{aligned}
$$

Assumptions (4) ensure that the right side in equality (20) is nonnegative; that is,

$$
\begin{aligned}
& \frac{a}{g^{\prime}} \Delta P+2 \frac{a^{\prime}+a}{g^{\prime}} \nabla u \cdot \nabla P \\
&+ \frac{a}{g^{\prime}}\left\{\left[1+\frac{a^{\prime}}{a}+\left(\frac{a^{\prime}}{a}\right)^{\prime}\right]|\nabla u|^{2}+\left(\frac{f}{a}\right)^{\prime}-\frac{f}{a}\right\} P \\
&-P_{t} \geq 0 \quad \text { in } \Omega \times(0, T) .
\end{aligned}
$$


Next, it follows from (1) and (10) that

$$
\begin{aligned}
\frac{\partial P}{\partial n} & =\mathrm{e}^{-u} u_{t} \frac{\partial u}{\partial n}-\mathrm{e}^{-u} \frac{\partial u_{t}}{\partial n}-\alpha \frac{a^{\prime}}{a^{2}} \frac{\partial u}{\partial n} \\
& =\mathrm{e}^{-u} u_{t} b-\mathrm{e}^{-u}\left(\frac{\partial u}{\partial n}\right)_{t}-\alpha \frac{a^{\prime}}{a^{2}} b \\
& =\mathrm{e}^{-u} u_{t} b-\mathrm{e}^{-u}(b(x, u, t))_{t}-\alpha \frac{a^{\prime}}{a^{2}} b \\
& =\left(b-b_{u}\right) \mathrm{e}^{-u} u_{t}-\mathrm{e}^{-u} b_{t}-\alpha \frac{a^{\prime}}{a^{2}} b \\
& =\left(b-b_{u}\right)\left(-P+\alpha \frac{1}{a}\right)-\mathrm{e}^{-u} b_{t}-\alpha \frac{a^{\prime}}{a^{2}} b \\
& =\left(b_{u}-b\right) P+\alpha \frac{a b-(a b)_{u}}{a^{2}}-\mathrm{e}^{-u} b_{t}
\end{aligned}
$$

We note that (6) implies

$$
\begin{aligned}
& \max _{\bar{\Omega}} P(x, 0)=\max _{\bar{\Omega}}\left\{-\mathrm{e}^{-u_{0}}\left(u_{0}\right)_{t}+\alpha \frac{1}{a\left(u_{0}\right)}\right\} \\
& =\max _{\bar{\Omega}}\left\{-\frac{\nabla \cdot\left(a\left(u_{0}\right) \nabla u_{0}\right)+f\left(u_{0}\right)}{\mathrm{e}^{u_{0}} g^{\prime}\left(u_{0}\right)}+\alpha \frac{1}{a\left(u_{0}\right)}\right\} \\
& =\max _{\bar{\Omega}}\left\{\frac{1}{a\left(u_{0}\right)}(\alpha\right. \\
& \left.\left.-\frac{a\left(u_{0}\right)\left[\nabla \cdot\left(a\left(u_{0}\right) \nabla u_{0}\right)+f\left(u_{0}\right)\right]}{\mathrm{e}^{u_{0}} g^{\prime}\left(u_{0}\right)}\right)\right\}=0 .
\end{aligned}
$$

There, (21)-(23), assumption (5) and the maximum principle [10] imply that the maximum of the function $P$ in $\bar{\Omega} \times[0, \mathrm{~T})$ is zero. In fact, if the function $P$ takes a positive maximum at point $\left(x_{0}, t_{0}\right) \in \partial \Omega \times(0, T)$, then we have

$$
P\left(x_{0}, t_{0}\right)>0,\left.\quad \frac{\partial P}{\partial n}\right|_{\left(x_{0}, t_{0}\right)}>0 .
$$

Using assumption (5) and the fact that $P\left(x_{0}, t_{0}\right)>0$, it follows from (22) that

$$
\left.\frac{\partial P}{\partial n}\right|_{\left(x_{0}, t_{0}\right)} \leq 0
$$

which contradicts the second inequality in (24). Hence, the maximum of the function $P$ in $\bar{\Omega} \times[0, T)$ is zero. Now, we have

$$
P \leq 0 \quad \text { in } \bar{\Omega} \times[0, T)
$$

that is,

$$
\frac{a(u)}{\mathrm{e}^{u}} u_{t} \geq \alpha
$$

At the point $x_{0} \in \bar{\Omega}$, where $u_{0}\left(x_{0}\right)=M_{0}$, integrating inequality (27) from 0 to $t$, we arrive at

$$
\int_{0}^{t} \frac{a(u)}{\mathrm{e}^{u}} u_{t} \mathrm{~d} t=\int_{M_{0}}^{u\left(x_{0}, t\right)} \frac{a(s)}{\mathrm{e}^{s}} \mathrm{~d} s \geq \alpha t .
$$

Inequality (28) and assumption (7) imply that $u(x, t)$ blows up in finite time $t=T$. Now, we let $t \rightarrow T$ in (28) to deduce

$$
T \leq \frac{1}{\alpha} \int_{M_{0}}^{+\infty} \frac{a(s)}{\mathrm{e}^{s}} \mathrm{~d} s .
$$

For each fixed $x \in \bar{\Omega}$, integrating inequality (27) from $t$ to $s(0<t<s<T)$, we get

$$
\begin{aligned}
H(u(x, t)) & \geq H(u(x, t))-H(u(x, s)) \\
& =\int_{u(x, t)}^{+\infty} \frac{a(s)}{\mathrm{e}^{s}} \mathrm{~d} s-\int_{u(x, s)}^{+\infty} \frac{a(s)}{\mathrm{e}^{s}} \mathrm{~d} s \\
& =\int_{u(x, t)}^{u(x, s)} \frac{a(s)}{\mathrm{e}^{s}} \mathrm{~d} s=\int_{t}^{s} \frac{a(u)}{\mathrm{e}^{u}} u_{t} \mathrm{~d} t \\
& \geq \alpha(s-t) .
\end{aligned}
$$

In the above inequality, letting $s \rightarrow T$, we obtain

$$
H(u(x, t)) \geq \alpha(T-t) .
$$

We note that $H$ is a strictly decreasing function. Hence,

$$
u(x, t) \leq H^{-1}(\alpha(T-t)) .
$$

The proof is complete.

\section{Global Solution}

In this section, we study what interactions among the four nonlinear mechanisms of (1) result in the global solution of (1). The main results of this section are formulated in the following theorem.

Theorem 2. Let $u(x, t)$ be a solution of problem (1). Assume that the following conditions (i)-(iv) are fulfilled.

(i) For $s \in \mathbb{R}_{+}$,

$$
\begin{aligned}
a(s)+a^{\prime}(s) & \leq 0, \\
f(s)+f^{\prime}(s) & \leq 0, \\
\left(\frac{a(s)}{g^{\prime}(s)}\right)^{\prime} & \leq 0 .
\end{aligned}
$$

(ii) $\operatorname{For}(x, s, t) \in \partial \Omega \times \mathbb{R}_{+} \times \mathbb{R}_{+}$,

$$
b(x, s, t)+b_{s}(x, s, t) \leq 0,
$$

$$
\begin{aligned}
a(s) b(x, s, t)+(a(s) b(x, s, t))_{s} & \leq 0, \\
b_{t}(x, s, t) & \leq 0 .
\end{aligned}
$$


(iii) Consider the following:

$$
\beta:=\max _{\bar{\Omega}}\left\{\frac{a\left(u_{0}\right)\left[\nabla \cdot\left(a\left(u_{0}\right) \nabla u_{0}\right)+f\left(u_{0}\right)\right]}{\mathrm{e}^{-u_{0}} g^{\prime}\left(u_{0}\right)}\right\}>0 .
$$

(iv) Consider the following:

$$
\int_{m_{0}}^{+\infty} \frac{a(s)}{\mathrm{e}^{-s}} \mathrm{~d} s=+\infty, \quad m_{0}:=\min _{\bar{\Omega}} u_{0}(x)
$$

Then $u(x, t)$ must be a global solution and

$$
u(x, t) \leq K^{-1}\left(\beta t+K\left(u_{0}(x)\right)\right),
$$

where

$$
K(y):=\int_{m_{0}}^{y} \frac{a(s)}{\mathrm{e}^{-s}} \mathrm{~d} s, \quad y \geq m_{0},
$$

and $K$ is the inverse function of $K$.

Proof. We consider an auxiliary function

$$
Q(x, t):=-\mathrm{e}^{u} u_{t}+\beta \frac{1}{a(u)} .
$$

Using the same reasoning process as that of (11)-(20), we obtain

$$
\begin{aligned}
\frac{a}{g^{\prime}} \Delta Q+2 \frac{a^{\prime}-a}{g^{\prime}} \nabla u \cdot \nabla Q \\
+\frac{a}{g^{\prime}}\left\{\left[1-\frac{a^{\prime}}{a}+\left(\frac{a^{\prime}}{a}\right)^{\prime}\right]|\nabla u|^{2}+\left(\frac{f}{a}\right)^{\prime}+\frac{f}{a}\right\} Q \\
-Q_{t} \\
=\beta \frac{1}{a g^{\prime}}\left(a+a^{\prime}\right)|\nabla u|^{2}+\frac{g^{\prime}}{a}\left(\frac{a}{g^{\prime}}\right)^{\prime} \mathrm{e}^{u}\left(u_{t}\right)^{2} \\
+\beta \frac{1}{a g^{\prime}}\left(f^{\prime}+f\right) .
\end{aligned}
$$

Assumptions (33) imply that the right side of (40) is nonpositive; that is,

$$
\begin{aligned}
\frac{a}{g^{\prime}} \Delta Q & +2 \frac{a^{\prime}-a}{g^{\prime}} \nabla u \cdot \nabla Q \\
+ & \frac{a}{g^{\prime}}\left\{\left[1-\frac{a^{\prime}}{a}+\left(\frac{a^{\prime}}{a}\right)^{\prime}\right]|\nabla u|^{2}+\left(\frac{f}{a}\right)^{\prime}+\frac{f}{a}\right\} Q \\
-Q_{t} & \leq 0 \quad \text { in } \Omega \times(0, T) .
\end{aligned}
$$

By (1) and (39), we get

$$
\begin{aligned}
\frac{\partial Q}{\partial n} & =-\mathrm{e}^{u} u_{t} \frac{\partial u}{\partial n}-\mathrm{e}^{u} \frac{\partial u_{t}}{\partial n}-\beta \frac{a^{\prime}}{a^{2}} \frac{\partial u}{\partial n} \\
& =-\mathrm{e}^{u} u_{t} b-\mathrm{e}^{u}\left(\frac{\partial u}{\partial n}\right)_{t}-\beta \frac{a^{\prime}}{a^{2}} b \\
& =-\mathrm{e}^{u} u_{t} b-\mathrm{e}^{u}(b(x, u, t))_{t}-\beta \frac{a^{\prime}}{a^{2}} b \\
& =-\left(b+b_{u}\right) \mathrm{e}^{u} u_{t}-\mathrm{e}^{u} b_{t}-\beta \frac{a^{\prime}}{a^{2}} b \\
& =\left(b+b_{u}\right)\left(Q-\beta \frac{1}{a}\right)-\mathrm{e}^{u} b_{t}-\beta \frac{a^{\prime}}{a^{2}} b \\
& =\left(b+b_{u}\right) Q-\beta \frac{a b+(a b)_{u}}{a^{2}}-\mathrm{e}^{u} b_{t}
\end{aligned}
$$

Assumption (35) implies

$$
\begin{aligned}
& \min _{\bar{\Omega}} Q(x, 0)=\min _{\bar{\Omega}}\left\{-\mathrm{e}^{u_{0}}\left(u_{0}\right)_{t}+\beta \frac{1}{a\left(u_{0}\right)}\right\} \\
& =\min _{\bar{\Omega}}\left\{-\frac{\nabla \cdot\left(a\left(u_{0}\right) \nabla u_{0}\right)+f\left(u_{0}\right)}{\mathrm{e}^{-u_{0}} g^{\prime}\left(u_{0}\right)}+\beta \frac{1}{a\left(u_{0}\right)}\right\} \\
& =\min _{\bar{\Omega}}\left\{\frac{1}{a\left(u_{0}\right)}(\beta\right. \\
& \left.\left.-\frac{a\left(u_{0}\right)\left[\nabla \cdot\left(a\left(u_{0}\right) \nabla u_{0}\right)+f\left(u_{0}\right)\right]}{\mathrm{e}^{-u_{0}} g^{\prime}\left(u_{0}\right)}\right)\right\}=0 .
\end{aligned}
$$

It follows from (41)-(43), (34), and the maximum principle that the minimum of $Q$ in $\bar{\Omega} \times[0, T)$ is zero. Hence, we have the following inequality:

$$
Q \geq 0 \text { in } \bar{\Omega} \times[0, T) ;
$$

that is

$$
\frac{a(u)}{\mathrm{e}^{-u}} u_{t} \leq \beta .
$$

For each fixed $x \in \bar{\Omega}$, we integrate (45) from 0 to $t$ to deduce

$$
\frac{1}{\beta} \int_{0}^{t} \frac{a(u)}{\mathrm{e}^{-u}} u_{t} \mathrm{~d} t=\frac{1}{\beta} \int_{u_{0}(x)}^{u(x, t)} \frac{a(s)}{\mathrm{e}^{-s}} \mathrm{~d} s \leq t .
$$

Inequality (46) and assumption (36) imply that $u$ must be a global solution. Furthermore, it follows from (45) that

$$
\begin{aligned}
K & (u(x, t))-K\left(u_{0}(x)\right) \\
& =\int_{m_{0}}^{u(x, t)} \frac{a(s)}{\mathrm{e}^{-s}} \mathrm{~d} s-\int_{m_{0}}^{u_{0}(x)} \frac{a(s)}{\mathrm{e}^{-s}} \mathrm{~d} s=\int_{u_{0}(x)}^{u(x, t)} \frac{a(s)}{\mathrm{e}^{-s}} \mathrm{~d} s \\
& =\int_{0}^{t} \frac{a(u)}{\mathrm{e}^{-u}} u_{t} \mathrm{~d} t \leq \beta t .
\end{aligned}
$$


Since $K$ is a strictly increasing function, we obtain

$$
u(x, t) \leq K^{-1}\left(\beta t+K\left(u_{0}(x)\right)\right) .
$$

The proof is complete.

\section{Applications}

When $a(u)=1$ and $b(x, u, t)=b(u)$, the results of Theorems 1-2 still hold. In this sense, our results extend and supplement those obtained in [21,22].

In the following, we give a few examples to demonstrate the applications of Theorems 1-2.

Example 3. Let $u$ be a solution of the following problem:

$$
\begin{aligned}
\left(\frac{u}{2}+\mathrm{e}^{u / 2}\right)_{t} & =\nabla \cdot\left(\mathrm{e}^{u / 2} \nabla u\right)+\mathrm{e}^{u} \quad \text { in } \Omega \times(0, T), \\
\frac{\partial u}{\partial n} & =\mathrm{e}^{(3 / 4)(u-2)}+\mathrm{e}^{u-2+t|x|^{2}}
\end{aligned}
$$

on $\partial \Omega \times(0, T)$,

$$
u(x, 0)=1+|x|^{2} \quad \text { in } \bar{\Omega},
$$

where $\Omega=\left\{x=\left.\left(x_{1}, x_{2}, x_{3}\right)|| x\right|^{2}=x_{1}^{2}+x_{2}^{2}+x_{3}^{2}<1\right\}$. We note

$$
\begin{aligned}
a(u) & =\mathrm{e}^{2 / u}, \\
g(u) & =\frac{u}{2}+\mathrm{e}^{u / 2}, \\
f(u) & =\mathrm{e}^{u}, \\
b(x, u, t) & =\mathrm{e}^{(3 / 4)(u-2)}+\mathrm{e}^{u-2+t|x|^{2}} .
\end{aligned}
$$

In order to calculate the constant $\alpha$, we set

$$
\omega=|x|^{2},
$$

and then $0 \leq \omega \leq 1$ and

$$
\begin{aligned}
\alpha & =\min _{\bar{\Omega}}\left\{\frac{a\left(u_{0}\right)\left[\nabla \cdot\left(a\left(u_{0}\right) \nabla u_{0}\right)+f\left(u_{0}\right)\right]}{\mathrm{e}^{u_{0}} g^{\prime}\left(u_{0}\right)}\right\} \\
& =\min _{\bar{\Omega}}\left\{\frac{2\left[6+2|x|^{2}+\mathrm{e}^{(1 / 2)\left(1+|x|^{2}\right)}\right]}{\mathrm{e}^{(1 / 2)\left(1+|x|^{2}\right)}+1}\right\} \\
& =\min _{0 \leq \omega \leq 1}\left\{\frac{2\left[6+2 \omega+\mathrm{e}^{(1 / 2)(1+\omega)}\right]}{\mathrm{e}^{(1 / 2)(1+\omega)}+1}\right\}=\frac{2(8+\mathrm{e})}{1+\mathrm{e}} .
\end{aligned}
$$

We can check that (4), (5), and (7) hold. It follows from Theorem 1 that $u(x, t)$ blows up in a finite time $T$ and

$$
\begin{aligned}
T & \leq \frac{1}{\alpha} \int_{M_{0}}^{+\infty} \frac{a(s)}{\mathrm{e}^{s}} \mathrm{~d} s=\frac{1+\mathrm{e}}{2(8+\mathrm{e})} \int_{2}^{+\infty} \mathrm{e}^{-s / 2} \mathrm{~d} s \\
& =\frac{1+\mathrm{e}}{\mathrm{e}(8+\mathrm{e})} \\
u(x, t) & \leq H^{-1}(\alpha(T-t))=2 \ln \frac{1+\mathrm{e}}{(8+\mathrm{e})(T-t)} .
\end{aligned}
$$

Example 4. Let $u$ be a solution of the following problem:

$$
\begin{aligned}
\left(u \mathrm{e}^{u}\right)_{t} & =\nabla \cdot\left(\frac{\mathrm{e}^{-u}}{1+u} \nabla u\right)+\mathrm{e}^{-u} \quad \text { in } \Omega \times(0, T), \\
\frac{\partial u}{\partial n} & =\mathrm{e}^{-2 u}+\mathrm{e}^{-u-t|x|^{2}} \quad \text { on } \partial \Omega \times(0, T), \\
u(x, 0) & =1+|x|^{2} \quad \text { in } \bar{\Omega},
\end{aligned}
$$

where $\Omega=\left\{x=\left.\left(x_{1}, x_{2}, x_{3}\right)|| x\right|^{2}=x_{1}^{2}+x_{2}^{2}+x_{3}^{2}<1\right\}$. Now we have

$$
\begin{aligned}
a(u) & =\frac{\mathrm{e}^{-u}}{1+u}, \\
g(u) & =u \mathrm{e}^{u}, \\
f(u) & =\mathrm{e}^{-u}, \\
b(x, u, t) & =\mathrm{e}^{-2 u}+\mathrm{e}^{-u-t|x|^{2}} .
\end{aligned}
$$

Setting

$$
\omega=|x|^{2}
$$

we get $0 \leq \omega \leq 1$ and

$$
\begin{aligned}
\beta & =\max _{\bar{\Omega}}\left\{\frac{a\left(u_{0}\right)\left[\nabla \cdot\left(a\left(u_{0}\right) \nabla u_{0}\right)+f\left(u_{0}\right)\right]}{\mathrm{e}^{-u_{0}} g^{\prime}\left(u_{0}\right)}\right\} \\
& =\max _{\bar{\Omega}}\left\{\frac{16-2|x|^{2}-3|x|^{4}}{\mathrm{e}^{2\left(1+|x|^{2}\right)}\left(2+|x|^{2}\right)^{4}}\right\} \\
& =\max _{0 \leq \omega \leq 1}\left\{\frac{16-2 \omega-3 \omega^{2}}{\mathrm{e}^{2(1+\omega)}(2+\omega)^{4}}\right\}=\mathrm{e}^{-2} .
\end{aligned}
$$

We can also check that (33), (34), and (36) hold. Hence, Theorem 2 implies $u$ must be a global solution and

$$
u(x, t) \leq K^{-1}\left(\beta t+K\left(u_{0}(x)\right)\right)=\left(2+|x|^{2}\right) \mathrm{e}^{t \mathrm{e}^{-2}}-1 .
$$

Remark 5. When the functions $a, b, f$, and $g$ are all exponential functions, Theorems 1 and 2 can be used. When not all of them are exponential functions, Theorems 1 and 2 can be used in some special cases.

\section{Competing Interests}

The author declares that there are no competing interests regarding the publication of this paper.

\section{Acknowledgments}

This work was supported by the National Natural Science Foundation of China (no. 61473180).

\section{References}

[1] P. Quittner and P. Souplet, Superlinear Parabolic Problems: BlowUp, Global Existence and Steady States, Birkhäuser Advanced Texts, Birkhäuser, Basel, Switzerland, 2007. 
[2] M. Marras and S. Vernier Piro, "On global existence and bounds for blow-up time in nonlinear parabolic problems with time dependent coefficients," Discrete and Continuous Dynamical Systems, vol. 2013, pp. 535-544, 2013.

[3] L. E. Payne, G. A. Philippin, and P. W. Schaefer, "Blow-up phenomena for some nonlinear parabolic problems," Nonlinear Analysis: Theory, Methods \& Applications, vol. 69, no. 10, pp. 3495-3502, 2008.

[4] S. H. Chen, "Global existence and nonexistence for some degenerate and quasilinear parabolic systems," Journal of Differential Equations, vol. 245, no. 4, pp. 1112-1136, 2008.

[5] S. H. Chen and K. MacDonald, "Global and blowup solutions for general quasilinear parabolic systems," Nonlinear Analysis: Real World Applications, vol. 14, no. 1, pp. 423-433, 2013.

[6] J. T. Ding and H. J. Hu, "Blow-up and global solutions for a class of nonlinear reaction diffusion equations under Dirichlet boundary conditions," Journal of Mathematical Analysis and Applications, vol. 433, no. 2, Article ID 19743, pp. 1718-1735, 2016.

[7] J. T. Ding and M. Wang, "Blow-up solutions, global existence, and exponential decay estimates for second order parabolic problems," Boundary Value Problems, vol. 2015, no. 168, 16 pages, 2015.

[8] L. L. Zhang, "Blow-up of solutions for a class of nonlinear parabolic equations," Zeitschrift für Analysis und Ihre Anwendungen, vol. 25, no. 4, pp. 479-486, 2006.

[9] H. Amann, "Quasilinear parabolic systems under nonlinear boundary conditions," Archive for Rational Mechanics and Analysis, vol. 92, no. 2, pp. 153-192, 1986.

[10] R. Sperb, Maximum Principles and Their Applications, Academic Press, New York, NY, USA, 1981.

[11] A. Friedman, Partial Differential Equation of Parabolic Type, Prentice-Hall, Englewood Cliffs, NJ, USA, 1964.

[12] J. Harada, "Non self-similar blow-up solutions to the heat equation with nonlinear boundary conditions," Nonlinear Analysis: Theory, Methods \& Applications, vol. 102, no. 2, pp. 36-83, 2014.

[13] J. Harada and K. Mihara, "Blow-up rate for radially symmetric solutions of some parabolic equations with nonlinear boundary conditions," Journal of Differential Equations, vol. 253, no. 5, pp. 1647-1663, 2012.

[14] Y. Liu, "Lower bounds for the blow-up time in a non-local reaction diffusion problem under nonlinear boundary conditions," Mathematical and Computer Modelling, vol. 57, no. 3-4, pp. 926931, 2013.

[15] Y. Liu, S. G. Luo, and Y. H. Ye, "Blow-up phenomena for a parabolic problem with a gradient nonlinearity under nonlinear boundary conditions," Computers \& Mathematics with Applications, vol. 65, no. 8, pp. 1194-1199, 2013.

[16] F. S. Li and J. L. Li, "Global existence and blow-up phenomena for nonlinear divergence form parabolic equations with inhomogeneous Neumann boundary conditions," Journal of Mathematical Analysis and Applications, vol. 385, no. 2, pp. 1005-1014, 2012.

[17] F. Liang, "Blow-up and global solutions for nonlinear reactiondiffusion equations with nonlinear boundary condition," Applied Mathematics and Computation, vol. 218, no. 8, pp. 3993-3999, 2011.

[18] L. E. Payne, G. A. Philippin, and S. Vernier Piro, "Blowup phenomena for a semilinear heat equation with nonlinear boundary condition, II," Nonlinear Analysis: Theory, Methods \& Applications, vol. 73, no. 4, pp. 971-978, 2010.
[19] J. Ding, X. Gao, and S. Li, "Global existence and blow-up problems for reaction diffusion model with multiple nonlinearities," Journal of Mathematical Analysis and Applications, vol. 343, no. 1, pp. 159-169, 2008.

[20] Y. W. Qi, M. X. Wang, and Z. J. Wang, "Existence and nonexistence of global solutions of diffusion systems with nonlinear boundary conditions," Proceedings of the Royal Society of Edinburgh-Section A: Mathematics, vol. 134, no. 6, pp. 11991217,2004

[21] H. L. Zhang, "Blow-up solutions and global solutions for nonlinear parabolic problems," Nonlinear Analysis: Theory, Methods \& Applications, vol. 69, no. 12, pp. 4567-4574, 2008.

[22] L. L. Zhang, N. Zhang, and L. X. Li, "Blow-up solutions and global existence for a kind of quasilinear reaction-diffusion equations," Zeitschrift für Analysis und ihre Anwendungen, vol. 33, no. 3, pp. 247-258, 2014. 


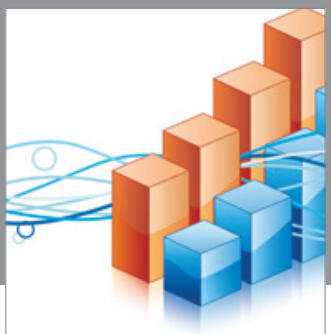

Advances in

Operations Research

vatem alat4

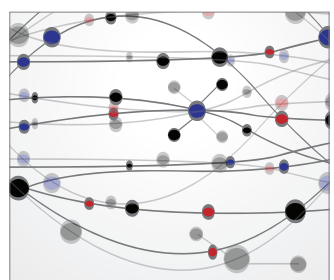

\section{The Scientific} World Journal
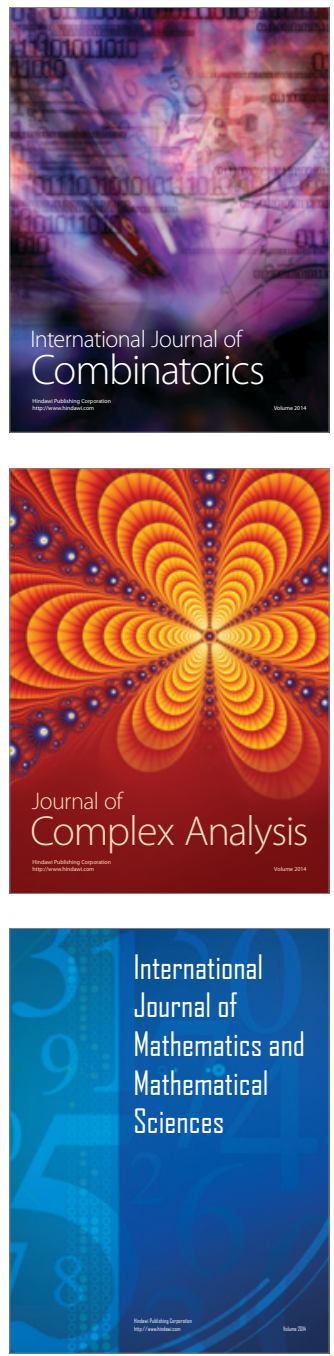
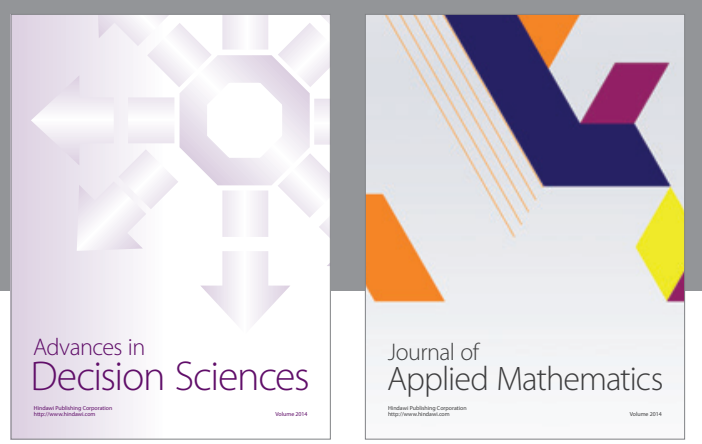

Algebra

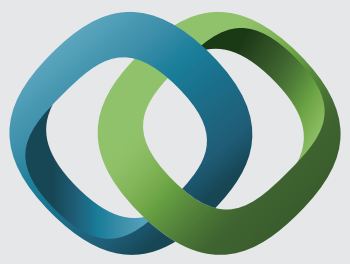

\section{Hindawi}

Submit your manuscripts at

http://www.hindawi.com
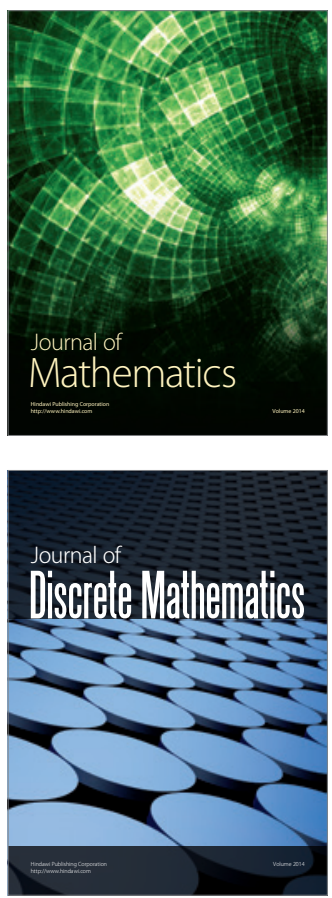

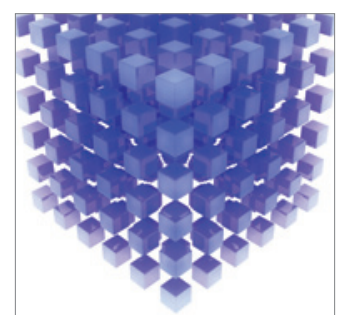

Mathematical Problems in Engineering
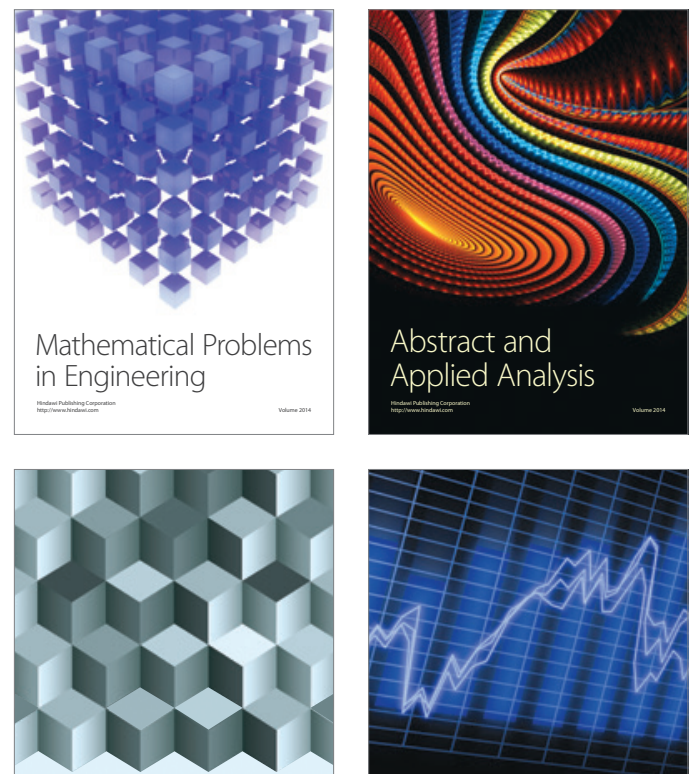

Journal of

Function Spaces

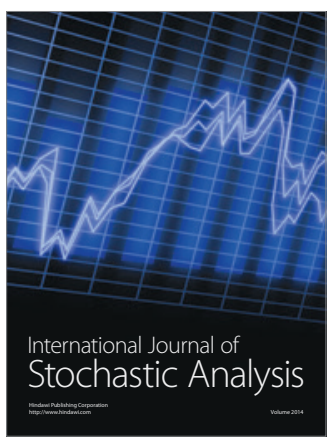

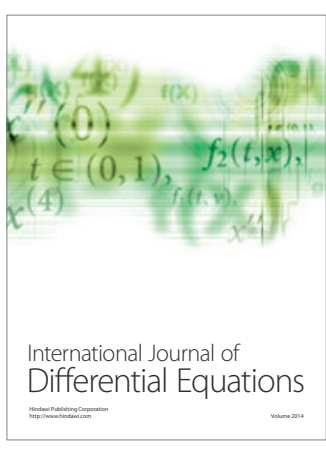
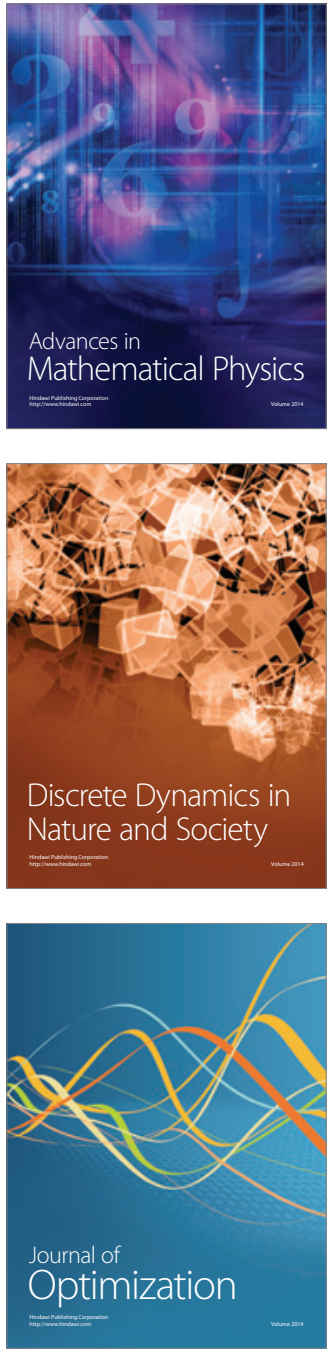